\title{
Orthogonal polynomials with respect to the sum of an arbitrary measure and a Bernstein-Szegö measure *
}

\author{
A. Cachafeiro ${ }^{\mathrm{a}}$, F. Marcellán ${ }^{\mathrm{b}}$ and C. Pérez ${ }^{\mathrm{c}}$ \\ ${ }^{a}$ Departamento de Matemática Aplicada I, E.T.S. Ingenieros Industriales, Universidad de Vigo, \\ 36280 Vigo, Spain \\ E-mail: acachafe@uvigo.es \\ ${ }^{\mathrm{b}}$ Departamento de Matemáticas, Escuela Politécnica Superior, Universidad Carlos III de Madrid, \\ 28911 Leganés, Spain \\ E-mail: pacomarc@ing.uc3m.es \\ ${ }^{\mathrm{c}}$ Departamento Didáctico de Matemáticas, I.E.S. Teis de Vigo, 36216 Vigo, Spain \\ E-mail: carlosp@edu.xunta.es
}

To Professor Dr. Mariano Gasca with occasion of his 60th anniversary.

\begin{abstract}
In the present paper we study the orthogonal polynomials with respect to a measure which is the sum of a finite positive Borel measure on $[0,2 \pi]$ and a Bernstein-Szegö measure. We prove that the measure sum belongs to the Szegö class and we obtain several properties about the norms of the orthogonal polynomials, as well as, about the coefficients of the expression which relates the new orthogonal polynomials with the Bernstein-Szegö polynomials. When the Bernstein-Szegö measure corresponds to a polynomial of degree one, we give a nice explicit algebraic expression for the new orthogonal polynomials.
\end{abstract}

Keywords: orthogonal polynomials, Bernstein-Szegö measure, Laguerre-Hahn affine functional.

Mathematics subject classifications (2000): 33C47, 42C05.

\section{Introduction}

It is well known [7] that polynomials orthogonal with respect to some positive Borel measure supported on the unit circle are related to Hermitian Toeplitz matrices. On the other hand, they are connected with the characteristic polynomials of principal submatrices of a structured upper Hessenberg matrix [8]. In the case of measures supported on the real line the Hessenberg matrix becomes a Jacobi matrix.

\footnotetext{
^ The research was supported by Dirección General de Investigación (Ministerio de Ciencia y Tecnología) of Spain under grant number BFM2000-0015, as well as BFM2003-06335-C03-C02.
} 
These polynomials are usually required for least squares data fitting. In particular, they are very useful in linear prediction theory of discrete stationary stochastic processes [7].

There are several situations where the Toeplitz matrices for certain given measures are explicitly known and one wants to determine a set of polynomials which are orthogonal with respect to a measure which is a combination of the given measures. In the case of measures supported in the real line, this problem has been introduced in [4].

In particular, in [1] this problem was studied for the sum of a measure $\mu$ supported on the unit circle and the normalized Lebesgue measure. The translation in terms of the entries of the resulting Toeplitz matrix means that we only perturb the main diagonal of the Toeplitz matrix associated with $\mu$ by adding a constant term. This fact is strongly connected with a method introduced by Pisarenko [9] for retrieving harmonics from a covariance function. It is based on a classical result by Carathéodory about the trigonometric moment problem. The relation with the "maximum entropy" spectral estimator and the effect of the addition of a noise component is analyzed there.

The generalization of the problem studied in [1] can be done in two different ways. The first one consists in the perturbation of the Toeplitz matrix $T(\mu)$ associated with $\mu$ by adding a finite number of moments of the second measure to the corresponding moments of $T(\mu)$. This approach has a numerical interest in perturbation theory for eigenvalues of Toeplitz matrices.

The second one has an analytical flavour. If $v$ is a positive Borel measure supported on the unit circle and $\left\{\varphi_{n}\right\}_{n=0}^{\infty}$ is the sequence of orthonormal polynomials with respect to $v$, i.e.

$$
\left\langle\varphi_{n}, \varphi_{m}\right\rangle_{\nu}=\int_{0}^{2 \pi} \varphi_{n}\left(\mathrm{e}^{\mathrm{i} \theta}\right) \overline{\varphi_{m}\left(\mathrm{e}^{\mathrm{i} \theta}\right)} \mathrm{d} \nu(\theta)=\delta_{m, n},
$$

then

$$
\mathrm{d} \nu_{k}=\frac{1}{\left|\varphi_{k}^{*}\left(\mathrm{e}^{\mathrm{i} \theta}\right)\right|^{2}} \frac{\mathrm{d} \theta}{2 \pi},
$$

with $\varphi_{k}^{*}(z)=z^{k} \overline{\varphi_{k}}\left(z^{-1}\right), k=1,2, \ldots$, is a sequence of positive Borel measures supported on the unit circle such that $v$ is the $*$-weak limit of the sequence $\left\{v_{k}\right\}$ [6].

Furthermore,

$$
\int_{0}^{2 \pi} P\left(\mathrm{e}^{\mathrm{i} \theta}\right) \mathrm{d} v_{k}(\theta)=\int_{0}^{2 \pi} P\left(\mathrm{e}^{\mathrm{i} \theta}\right) \mathrm{d} v(\theta),
$$

for every polynomial $P$ of degree at most $k$. The relation with numerical quadrature on the unit circle has been pointed out by several authors [3]. Thus, it seems to be natural to consider the sequence of measures $\mathrm{d} \mu_{k}=\mathrm{d} \mu+\mathrm{d} v_{k}$ and the corresponding sequences of orthogonal polynomials. A varying orthogonality appears and thus we are interested to analyze the sequence of monic polynomials associated with the measure $\mathrm{d} \mu_{k}$ for a fixed $k$. 
Taking into account the characterization of monic orthogonal polynomials as extremal polynomials, which can be read as a linear backward predictor in linear prediction theory, the aim of our contribution is to present a constructive approach to the sequence of monic polynomials orthogonal with respect to $\mathrm{d} \mu_{k}$ in terms of monic polynomials orthogonal with respect to $\mathrm{d} \mu$.

Notice that the sequence of polynomials orthonormal with respect to $v_{k}$ is known as Bernstein-Szegö sequence. They are explicitly given by $z^{n-k} \varphi_{k}(z)$ for $n \geqslant k$.

The structure of the paper is as follows.

In section 2 we obtain some properties concerning the measure $\tilde{\mu}$, which is the sum of a positive finite Borel measure $\mu$ and a Bernstein-Szegö measure $v$. We prove that $\tilde{\mu}$ belongs to the Szegö class and we obtain several relations between the norms of the sequence of monic orthogonal polynomials (SMOP) with respect to the three measures. We also deduce some properties about the coefficients of the expression which relates the SMOP with respect to measures $\tilde{\mu}$ and $\nu$.

In section 3 we study some properties of the Carathéodory function of the measure $\tilde{\mu}$. Remember that the Carathéodory function of a measure $\mu$ is defined as

$$
\mathbb{F}(z)=\frac{1}{2 \pi} \int_{0}^{2 \pi} \frac{\mathrm{e}^{\mathrm{i} \theta}+z}{\mathrm{e}^{\mathrm{i} \theta}-z} \mathrm{~d} \mu(\theta) .
$$

In section 4 we consider that the measure $\mu$ is a polynomial modification of the Lebesgue measure. In this case we obtain some interesting properties for the SMOP with respect to the measure sum.

Finally, in the last section, we assume that the Bernstein-Szegö measure is

$$
\mathrm{d} \nu(\theta)=\frac{1}{\left|A_{1}\left(\mathrm{e}^{\mathrm{i} \theta}\right)\right|^{2}} \frac{\mathrm{d} \theta}{2 \pi}
$$

with $A_{1}(z)$ a polynomial of degree one. In this case we give an explicit expression for the SMOP related to $\tilde{\mu}$.

\section{Orthogonality with respect to $\tilde{\mu}=\mu+v$, with $v$ a Bernstein-Szegö measure}

We consider the following measure

$$
\mathrm{d} \tilde{\mu}=\mathrm{d} \mu+\mathrm{d} \nu,
$$

where

$$
\mathrm{d} \nu=\frac{1}{\left|A_{k}\left(\mathrm{e}^{\mathrm{i} \theta}\right)\right|^{2}} \frac{\mathrm{d} \theta}{2 \pi} \quad \text { and } \quad A_{k}(z)=\sum_{i=0}^{k} \overline{a_{k-i}} z^{i}
$$

is a polynomial of degree $k$, such that $A_{k}(z) \neq 0$ in $\overline{\mathbb{D}}=\{z \in \mathbb{C}:|z| \leqslant 1\}$.

In the sequel we denote by $\left\{\phi_{n}\right\}_{n=0}^{\infty}$ the $\operatorname{SMOP}(\mu)$, by $\left\{\psi_{n}\right\}_{n=0}^{\infty}$ the $\operatorname{SMOP}(v)$ and by $\left\{\tilde{\phi}_{n}\right\}_{n=0}^{\infty}$ the $\operatorname{SMOP}(\tilde{\mu})$. For every Borel positive and finite measure $\mu$ on $[0,2 \pi]$ it is very well known (see [6]) that the sequence of the norms of the SMOP is decreasing. 
This fact is a consequence of the extremal property of the norms of the SMOP. Therefore $\left\{\left\|\phi_{n}\right\|_{\mu}\right\}_{n=0}^{\infty}$ is decreasing and hence there exists $\lim _{n \rightarrow \infty}\left\|\phi_{n}\right\|_{\mu}^{2}=m(\mu) \geqslant 0$.

The measure $\mu$ belongs to the Szegö class $\mathbb{S}$ if and only if $\int_{0}^{2 \pi} \log \mu^{\prime}(\theta) \mathrm{d} \theta>-\infty$, where we denote by $\mu^{\prime}$ the Radon-Nikodym derivative of $\mu$ with respect to the Lebesgue measure. The measures in the Szegö class can be characterized by different equivalent conditions (see $[6,10])$. If $\mu \in \mathbb{S}$, then the Szegö function is

$$
\Pi(z ; \mu)=\exp \left(\frac{-1}{4 \pi} \int_{0}^{2 \pi} \frac{\mathrm{e}^{\mathrm{i} \theta}+z}{\mathrm{e}^{\mathrm{i} \theta}-z} \log \mu^{\prime}(\theta) \mathrm{d} \theta\right),
$$

where $\mu^{\prime}$ is defined as above. When the measure belongs to the Szegö class $\mathbb{S}$ then the preceding limit, $m(\mu)$, is positive.

In the sequel we denote the limit of the norms of the SMOP in the following way:

$$
\lim _{n \rightarrow \infty}\left\|\phi_{n}\right\|_{\mu}^{2}=m(\mu), \quad \lim _{n \rightarrow \infty}\left\|\psi_{n}\right\|_{v}^{2}=m(v), \quad \text { and } \quad \lim _{n \rightarrow \infty}\left\|\tilde{\phi}_{n}\right\|_{\tilde{\mu}}^{2}=m(\tilde{\mu}) .
$$

Since for $n \geqslant k \psi_{n}(z)=1 / a_{k} z^{n-k} A_{k}^{*}(z)$, then $\left\|\psi_{n}(z)\right\|_{v}^{2}=1 /\left|a_{k}\right|^{2}$ for $n \geqslant k$, and therefore $m(v)=1 /\left|a_{k}\right|^{2}$.

Next we present some relations between the norms of the above polynomials.

Proposition 1. It holds that

(i) For $n \geqslant 0$

$$
m(\mu)+\frac{1}{\left|a_{k}\right|^{2}} \leqslant\left\|\tilde{\phi}_{n}\right\|_{\tilde{\mu}}^{2} \leqslant\left\|\psi_{n}\right\|_{\mu}^{2}+c_{0}(v),
$$

where $c_{0}(v)=\int_{0}^{2 \pi} \mathrm{d} v(\theta)$.

In particular, for $n \geqslant k$ we get

$$
m(\mu)+\frac{1}{\left|a_{k}\right|^{2}} \leqslant\left\|\tilde{\phi}_{n}\right\|_{\tilde{\mu}}^{2} \leqslant \frac{1}{\left|a_{k}\right|^{2}}\left(1+\left\|A_{k}^{*}\right\|_{\mu}^{2}\right) .
$$

(ii) The following relations between the norms are valid for every $n \geqslant 0$

$$
\begin{aligned}
& \text { (a) }\left\|\phi_{n}\right\|_{\mu}^{2} \leqslant\left\|\tilde{\phi}_{n}\right\|_{\mu}^{2} \leqslant\left\|\psi_{n}\right\|_{\mu}^{2}, \\
& \text { (b) }\left\|\psi_{n}\right\|_{\nu}^{2} \leqslant\left\|\tilde{\phi}_{n}\right\|_{\nu}^{2} \leqslant\left\|\phi_{n}\right\|_{\nu}^{2} .
\end{aligned}
$$

Proof. We take into account the minimal property of the norms of monic orthogonal polynomials.

(i) To get (2), we consider the following inequalities, which are valid for every $n \geqslant 0$,

$$
\begin{aligned}
& \left\|\tilde{\phi}_{n}\right\|_{\tilde{\mu}}^{2} \leqslant\left\|\psi_{n}\right\|_{\tilde{\mu}}^{2}=\left\|\psi_{n}\right\|_{\mu}^{2}+\left\|\psi_{n}\right\|_{\nu}^{2} \leqslant\left\|\psi_{n}\right\|_{\mu}^{2}+c_{0}(v) \quad \text { and } \\
& \left\|\tilde{\phi}_{n}\right\|_{\tilde{\mu}}^{2}=\left\|\tilde{\phi}_{n}\right\|_{\mu}^{2}+\left\|\tilde{\phi}_{n}\right\|_{\nu}^{2} \geqslant\left\|\phi_{n}\right\|_{\mu}^{2}+\left\|\psi_{n}\right\|_{\nu}^{2} \geqslant m(\mu)+\frac{1}{\left|a_{k}\right|^{2}} .
\end{aligned}
$$


Since, for $n \geqslant k,\left\|\psi_{n}\right\|_{v}^{2}=1 /\left|a_{k}\right|^{2}$, then

$$
m(\mu)+\frac{1}{\left|a_{k}\right|^{2}} \leqslant\left\|\tilde{\phi}_{n}\right\|_{\tilde{\mu}}^{2} \leqslant\left\|\psi_{n}\right\|_{\mu}^{2}+\frac{1}{\left|a_{k}\right|^{2}},
$$

which yields (3).

(ii) To obtain (4) it is enough to consider the following inequalities

$$
\left\|\phi_{n}\right\|_{\mu}^{2}+\left\|\psi_{n}\right\|_{\nu}^{2} \leqslant\left\|\tilde{\phi}_{n}\right\|_{\mu}^{2}+\left\|\psi_{n}\right\|_{\nu}^{2} \leqslant\left\|\tilde{\phi}_{n}\right\|_{\mu}^{2}+\left\|\tilde{\phi}_{n}\right\|_{\nu}^{2} \leqslant\left\|\psi_{n}\right\|_{\mu}^{2}+\left\|\psi_{n}\right\|_{\nu}^{2} .
$$

Proceeding in a similar way,

$$
\left\|\tilde{\phi}_{n}\right\|_{\mu}^{2}+\left\|\psi_{n}\right\|_{v}^{2} \leqslant\left\|\tilde{\phi}_{n}\right\|_{\mu}^{2}+\left\|\tilde{\phi}_{n}\right\|_{v}^{2} \leqslant\left\|\phi_{n}\right\|_{\mu}^{2}+\left\|\phi_{n}\right\|_{v}^{2} \leqslant\left\|\tilde{\phi}_{n}\right\|_{\mu}^{2}+\left\|\phi_{n}\right\|_{v}^{2},
$$

and we get (5).

Corollary 1. $\tilde{\mu}$ belongs to the Szegö class $\mathbb{S}$ and the inverse of the weight of orthogonality is integrable, that is,

(i) $\tilde{\mu} \in \mathbb{S}$.

(ii) $\left(1 / \tilde{\mu}^{\prime}(\theta)\right) \in L^{1}[0,2 \pi]$, where $\tilde{\mu}^{\prime}$ denotes the Radon-Nikodym derivative of $\tilde{\mu}$ with respect to the Lebesgue measure.

Proof. (i) If we take limits in (3) we get

$$
\frac{1}{\left|a_{k}\right|^{2}}+m(\mu) \leqslant m(\tilde{\mu}) .
$$

Therefore $m(\tilde{\mu})>0$ and hence $\tilde{\mu} \in \mathbb{S}$.

(ii) Since

$$
\mathrm{d} \tilde{\mu}(\theta)=\mathrm{d} \mu(\theta)+\frac{1}{2 \pi\left|A_{k}\left(\mathrm{e}^{\mathrm{i} \theta}\right)\right|^{2}} \mathrm{~d} \theta,
$$

then

$$
\tilde{\mu}^{\prime}(\theta)=\mu^{\prime}(\theta)+\frac{1}{2 \pi\left|A_{k}\left(\mathrm{e}^{\mathrm{i} \theta}\right)\right|^{2}}, \quad \text { a.e. }
$$

Therefore

$$
\frac{1}{\tilde{\mu}^{\prime}(\theta)}=\frac{2 \pi\left|A_{k}\left(\mathrm{e}^{\mathrm{i} \theta}\right)\right|^{2}}{1+2 \pi\left|A_{k}\left(\mathrm{e}^{\mathrm{i} \theta}\right)\right|^{2} \mu^{\prime}(\theta)} \leqslant 2 \pi\left|A_{k}\left(\mathrm{e}^{\mathrm{i} \theta}\right)\right|^{2}, \quad \text { a.e., }
$$

which implies that $\left(1 / \tilde{\mu}^{\prime}(\theta)\right) \in L^{1}[0,2 \pi]$.

Proposition 2. The following statements hold

$$
\begin{aligned}
& \text { (i) }\left\|\tilde{\phi}_{n}-\psi_{n}\right\|_{\tilde{\mu}}^{2} \leqslant\left\|\psi_{n}\right\|_{\mu}^{2}-m(\mu), \\
& \text { (ii) }\left\|\tilde{\phi}_{n}-\phi_{n}\right\|_{\tilde{\mu}}^{2} \leqslant\left\|\phi_{n}\right\|_{\nu}^{2}-\frac{1}{\left|a_{k}\right|^{2}} .
\end{aligned}
$$


Proof. (i) Taking into account that the polynomials $\tilde{\phi}_{n}, \psi_{n}$, and $\phi_{n}$ are monic we compute

$$
\begin{aligned}
\left\|\tilde{\phi}_{n}-\psi_{n}\right\|_{\tilde{\mu}}^{2} & =\left\langle\tilde{\phi}_{n}-\psi_{n}, \tilde{\phi}_{n}-\psi_{n}\right\rangle_{\tilde{\mu}}=-\left\langle\psi_{n}, \tilde{\phi}_{n}-\psi_{n}\right\rangle_{\tilde{\mu}} \\
& =\left\|\psi_{n}\right\|_{\tilde{\mu}}^{2}-\left\|\tilde{\phi}_{n}\right\|_{\tilde{\mu}}^{2}=\left\|\psi_{n}\right\|_{\mu}^{2}+\left\|\psi_{n}\right\|_{\nu}^{2}-\left\|\tilde{\phi}_{n}\right\|_{\tilde{\mu}}^{2} .
\end{aligned}
$$

Since $\left\|\phi_{n}\right\|_{\mu}^{2}+\left\|\psi_{n}\right\|_{\nu}^{2} \leqslant\left\|\tilde{\phi}_{n}\right\|_{\mu}^{2}+\left\|\tilde{\phi}_{n}\right\|_{\nu}^{2}=\left\|\tilde{\phi}_{n}\right\|_{\tilde{\mu}}^{2}$, then

$$
\left\|\tilde{\phi}_{n}-\psi_{n}\right\|_{\tilde{\mu}}^{2} \leqslant\left\|\psi_{n}\right\|_{\mu}^{2}-\left\|\phi_{n}\right\|_{\mu}^{2} \leqslant\left\|\psi_{n}\right\|_{\mu}^{2}-m(\mu) .
$$

(ii) Proceeding in a similar way we have

$$
\begin{aligned}
\left\|\tilde{\phi}_{n}-\phi_{n}\right\|_{\tilde{\mu}}^{2} & =\left\langle\tilde{\phi}_{n}-\phi_{n}, \tilde{\phi}_{n}-\phi_{n}\right\rangle_{\tilde{\mu}}=-\left\langle\phi_{n}, \tilde{\phi}_{n}-\left.\phi_{n}\right|_{\tilde{\mu}}\right. \\
& =\left\|\phi_{n}\right\|_{\tilde{\mu}}^{2}-\left\|\tilde{\phi}_{n}\right\|_{\tilde{\mu}}^{2}=\left\|\phi_{n}\right\|_{\mu}^{2}+\left\|\phi_{n}\right\|_{v}^{2}-\left\|\tilde{\phi}_{n}\right\|_{\tilde{\mu}}^{2} .
\end{aligned}
$$

Now, applying again that $\left\|\phi_{n}\right\|_{\mu}^{2}+\left\|\psi_{n}\right\|_{\nu}^{2} \leqslant\left\|\tilde{\phi}_{n}\right\|_{\tilde{\mu}}^{2}$, we get

$$
\left\|\tilde{\phi}_{n}-\phi_{n}\right\|_{\tilde{\mu}}^{2} \leqslant\left\|\phi_{n}\right\|_{v}^{2}-\left\|\psi_{n}\right\|_{v}^{2} \leqslant\left\|\phi_{n}\right\|_{v}^{2}-\frac{1}{\left|a_{k}\right|^{2}} .
$$

If we write

$$
\tilde{\phi}_{n}(z)=\psi_{n}(z)+\sum_{j=0}^{n-1} \beta_{n, j} \psi_{j}(z), \quad \beta_{n, j} \in \mathbb{C},
$$

we are going to study the coefficients $\beta_{n, j}$.

Proposition 3. The following statements hold:

(i) For every $n \geqslant 1$, the coefficients $\beta_{n, j}$ with $j=0,1, \ldots, n-1$ in (8) are bounded by

$$
\left|\beta_{n, j}\right| \leqslant\left(c_{0}(\mu)+c_{0}(v)\right)\left|a_{k}\right| .
$$

(ii) The sequences of coefficients $\left\{\beta_{n, n-j}\right\}_{j \leqslant n-k}$ are convergent.

(iii) For each $n>k$ it holds that

$$
\left|\tilde{\phi}_{n}^{(j)}(0)\right| \leqslant\left(c_{0}(\mu)+c_{0}(v)\right)\left|a_{k}\right| \sum_{i=j}^{j+k}\left|\psi_{i}^{(j)}(0)\right|, \quad \text { for every } j=0,1, \ldots, n-k-1,
$$

and therefore, for $n>2 k$,

$$
\frac{\left|\tilde{\phi}_{n}^{(j)}(0)\right|}{j !} \leqslant\left(c_{0}(\mu)+c_{0}(v)\right) \sum_{i=0}^{k}\left|a_{i}\right|, \quad \text { for } k \leqslant j \leqslant n-k-1 .
$$


Proof. (i) From (8) we get

$$
\left\langle\tilde{\phi}_{n}(z), \psi_{j}(z)\right\rangle_{v}=\beta_{n, j}\left\|\psi_{j}\right\|_{v}^{2}, \quad 0 \leqslant j \leqslant n-1 .
$$

Then

$$
\left|\beta_{n, j}\right|=\frac{\left|\left\langle\tilde{\phi}_{n}(z), \psi_{j}(z)\right\rangle_{v}\right|}{\left\|\psi_{j}\right\|_{\nu}^{2}} \leqslant \frac{\left\|\tilde{\phi}_{n}\right\|_{v}}{\left\|\psi_{j}\right\|_{\nu}} \leqslant\left\|\tilde{\phi}_{n}\right\|_{\nu}\left|a_{k}\right| .
$$

Moreover, since $\left\|\tilde{\phi}_{n}\right\|_{\nu} \leqslant\left\|\tilde{\phi}_{n}\right\|_{\tilde{\mu}}$ then

$$
\left|\beta_{n, j}\right| \leqslant\left\|\tilde{\phi}_{n}(z)\right\|_{\tilde{\mu}}\left|a_{k}\right| \leqslant c_{0}(\tilde{\mu})\left|a_{k}\right|=\left(c_{0}(\mu)+c_{0}(v)\right)\left|a_{k}\right|, \quad j=0,1, \ldots, n-1,
$$

and (9) holds.

(ii) On the other hand, for every $j$ such that $j \leqslant n-k$, we have

$$
\begin{aligned}
\beta_{n, n-j} & =\frac{\left\langle\tilde{\phi}_{n}(z), \psi_{n-j}(z)\right\rangle_{v}}{\left\|\psi_{n-j}\right\|_{v}^{2}}=\frac{\left\langle z^{n}+\sum_{i=1}^{j}\left(\tilde{\phi}_{n}^{(n-i)}(0) /(n-i) !\right) z^{n-i}, \psi_{n-j}(z)\right\rangle_{v}}{\left\|\psi_{n-j}\right\|_{v}^{2}} \\
& =\sum_{i=0}^{j} \frac{\left\langle\left(\tilde{\phi}_{n}^{(n-i)}(0) /(n-i) !\right) z^{n-i}, z^{n-j-k} A_{k}^{*}(z)\right\rangle_{v}}{a_{k}\left\|\psi_{n-j}\right\|_{v}^{2}}=\sum_{i=0}^{j} \frac{\tilde{\phi}_{n}^{(n-i)}(0)}{(n-i) !} \frac{\left\langle z^{j+k-i}, A_{k}^{*}(z)\right\rangle_{v}}{a_{k}\left\|\psi_{n-j}\right\|_{v}^{2}} .
\end{aligned}
$$

Therefore, if we denote by $\mathcal{A}_{i}=\lim _{n \rightarrow \infty}\left(\tilde{\phi}_{n}^{(n-i)}(0) /(n-i) !\right), i=1,2, \ldots, j$, then we get

$$
\lim _{n \rightarrow \infty} \beta_{n, n-j}=\frac{1}{m(v)} \sum_{i=0}^{j} \mathcal{A}_{i}\left\langle z^{j+k-i}, A_{k}^{*}\right\rangle_{\nu}
$$

(iii) Since $\psi_{n}^{(j)}(0)=0, \forall n>k+j$ with $j \geqslant 0$, from relation (8), we have for every $j$ such that $0 \leqslant j \leqslant n-k-1$

$$
\tilde{\phi}_{n}^{(j)}(0)=\sum_{i=0}^{k} \beta_{n, j+i} \psi_{j+i}^{(j)}(0)
$$

Now applying (9) we get

$$
\left|\tilde{\phi}_{n}^{(j)}(0)\right| \leqslant \sum_{i=0}^{k}\left|\beta_{n, j+i}\right|\left|\psi_{j+i}^{(j)}(0)\right| \leqslant\left(c_{0}(\mu)+c_{0}(\nu)\right)\left|a_{k}\right| \sum_{i=0}^{k}\left|\psi_{j+i}^{(j)}(0)\right|,
$$

which yields (10).

For $n>2 k$, to get (11) it is enough to consider (10) and take into account that for each $j$ such that $k \leqslant j \leqslant n-k-1$ it holds $\psi_{j+s}^{(j)}(0)=j ! a_{k-s} / a_{k}$ for every $s$ such that $0 \leqslant s \leqslant k$. 
Corollary 2. The coefficients of (8) satisfy

$$
\text { (i) } \sum_{j=0}^{n-1}\left|\beta_{n, j}\right|^{2} \leqslant\left|a_{k}\right|^{2}\left(\left\|\tilde{\phi}_{n}\right\|_{\tilde{\mu}}^{2}-\left\|\psi_{n}\right\|_{\nu}^{2}\right) \text {. }
$$

For $n \geqslant k$ the above expression becomes

$$
\begin{gathered}
\sum_{j=0}^{n-1}\left|\beta_{n, j}\right|^{2} \leqslant\left|a_{k}\right|^{2}\left\|A_{k}^{*}\right\|_{\mu}^{2} . \\
\text { (ii) } \sum_{j=0}^{n-1}\left|\beta_{n, j}\right|^{2} \leqslant\left|a_{k}\right|^{2}\left(c_{0}(\mu)+c_{0}(v)-\frac{1}{\left|a_{k}\right|^{2}}\right) .
\end{gathered}
$$

Proof. (i) If we take $v$ norms in (8), then we have

$$
\left\|\tilde{\phi}_{n}-\psi_{n}\right\|_{\nu}^{2}=\sum_{j=0}^{n-1}\left|\beta_{n, k}\right|^{2}\left\|\psi_{k}\right\|_{v}^{2} \geqslant m(v) \sum_{j=0}^{n-1}\left|\beta_{n, j}\right|^{2}=\frac{1}{\left|a_{k}\right|^{2}} \sum_{j=0}^{n-1}\left|\beta_{n, j}\right|^{2} .
$$

Besides, since

$$
\left\|\tilde{\phi}_{n}-\psi_{n}\right\|_{v}^{2}=\left\langle\tilde{\phi}_{n}-\psi_{n}, \tilde{\phi}_{n}-\psi_{n}\right\rangle_{v}=\left\langle\tilde{\phi}_{n}-\psi_{n}, \tilde{\phi}_{n}\right\rangle_{v}=\left\|\tilde{\phi}_{n}\right\|_{v}^{2}-\left\|\psi_{n}\right\|_{v}^{2},
$$

we obtain (12), because

$$
\sum_{j=0}^{n-1}\left|\beta_{n, j}\right|^{2} \leqslant\left|a_{k}\right|^{2}\left(\left\|\tilde{\phi}_{n}\right\|_{\nu}^{2}-\left\|\psi_{n}\right\|_{\nu}^{2}\right) \leqslant\left|a_{k}\right|^{2}\left(\left\|\tilde{\phi}_{n}\right\|_{\tilde{\mu}}^{2}-\left\|\psi_{n}\right\|_{\nu}^{2}\right) .
$$

Since $\left\|\tilde{\phi}_{n}\right\|_{\tilde{\mu}}^{2} \leqslant\left\|\psi_{n}\right\|_{\tilde{\mu}}^{2}$, from (12) for every $n$ we get

$$
\sum_{j=0}^{n-1}\left|\beta_{n, j}\right|^{2} \leqslant\left|a_{k}\right|^{2}\left(\left\|\psi_{n}\right\|_{\tilde{\mu}}^{2}-\left\|\psi_{n}\right\|_{\nu}^{2}\right),
$$

and since $\left\|\psi_{n}\right\|_{\tilde{\mu}}^{2}=\left\|\psi_{n}\right\|_{\mu}^{2}+\left\|\psi_{n}\right\|_{\nu}^{2}$, we deduce that $\sum_{j=0}^{n-1}\left|\beta_{n, j}\right|^{2} \leqslant\left|a_{k}\right|^{2}\left\|\psi_{n}\right\|_{\mu}^{2}$ and for $n \geqslant k$ (13) follows. from (12)

(ii) Taking into account that for $n \geqslant 0,\left\|\tilde{\phi}_{n}\right\|_{\tilde{\mu}}^{2} \leqslant c_{0}(\tilde{\mu})$ and $\left\|\psi_{n}\right\|_{\nu}^{2} \geqslant m(v)$,

$$
\sum_{j=0}^{n-1}\left|\beta_{n, j}\right|^{2} \leqslant\left|a_{k}\right|^{2}\left(c_{0}(\tilde{\mu})-m(v)\right)=\left|a_{k}\right|^{2}\left(c_{0}(\mu)+c_{0}(v)-\frac{1}{\left|a_{k}\right|^{2}}\right),
$$

and hence we get (14). 


\section{The Laguerre-Hahn affine character of the measure sum}

We denote by $£_{\nu}$ the linear functional associated with the Bernstein-Szegö measure

$$
\mathrm{d} \nu(\theta)=\frac{1}{2 \pi\left|A_{k}\left(\mathrm{e}^{\mathrm{i} \theta}\right)\right|^{2}} \mathrm{~d} \theta,
$$

and by $\mathbb{F}_{v}$ its Carathéodory function. Let us consider the linear functional of second kind, $L_{v}$ (see [6]).

It is well known that the Szegö function of $£_{v}$ is given by $\Pi\left(z ; £_{v}\right)=A_{k}(z) / \bar{a}_{k}$, and the Szegö function of $L_{v}$ is given in a similar way by $\Pi\left(z ; L_{v}\right)=B_{k}(z)$, with $B_{k}$ a polynomial of degree $k$. Since

$$
\mathbb{F}_{v}(z)=\frac{\Pi\left(z ; L_{v}\right)}{\Pi\left(0 ; L_{v}\right)} \frac{\Pi\left(z ; £_{v}\right)}{\Pi\left(0 ; £_{v}\right)},
$$

then the Carathéodory function $\mathbb{F}_{v}$ is rational. Therefore it is well known that $£_{v}$ is a Laguerre-Hahn affine linear functional of second degree (see [2]).

We recall the following definition:

Definition 1 [2]. A quasi-definite and Hermitian linear functional is said to be a Laguerre-Hahn affine linear functional if there exist polynomials $A, B$, and $H$, with $A \neq 0$, such that the formal series $\mathbb{G}(z)$ and its formal derivative $\mathbb{G}^{\prime}(z)$ satisfy

$$
A(z) \mathbb{G}^{\prime}(z)+B(z) \mathbb{G}(z)+H(z)=0,
$$

where

$$
\mathbb{G}(z)=\frac{\mathbb{F}(z)+\overline{\mathbb{F}}(1 / z)}{2} .
$$

Notice that $\mathbb{F}$ is rational if and only if $\mathbb{G}$ is rational.

Besides, the following characterization is known.

Proposition 4. Let $\mathcal{L}$ be a quasi-definite and Hermitian linear functional with a Carathéodory function $\mathbb{F}$ which is a non rational formal series. The following conditions are equivalent:

(i) $\mathcal{L}$ is Laguerre-Hahn affine and equation (15) is satisfied

$$
A(z) \mathbb{G}^{\prime}(z)+B(z) \mathbb{G}(z)+H(z)=0 \text { with } A \neq 0 .
$$

(ii) There exist polynomials $\mathcal{A}, \mathcal{B}$, and $\mathcal{H}$ such that $\mathcal{A} \neq 0$,

$$
z^{2} \overline{\mathcal{A}}\left(\frac{1}{z}\right) \mathcal{B}(z)+\mathcal{A}(z) \overline{\mathcal{B}}\left(\frac{1}{z}\right)=0, \quad \text { and } \quad \mathcal{A}(z) \mathbb{F}^{\prime}(z)+\mathcal{B}(z) \mathbb{F}(z)+\mathcal{H}(z)=0 .
$$

Proof. See [2]. 
In what follows we denote by $£$ the linear functional associated with the measure $\mu$ and by $\widetilde{£}$ the corresponding to $\tilde{\mu}=\mu+v$.

Proposition 5. $\widetilde{£}$ satisfies the following properties.

(i) If the linear functional $£$ belongs to the Laguerre-Hahn affine class, then $\widetilde{£}$ also belongs to the Laguerre-Hahn affine class.

(ii) If the linear functional $£$ is of second degree, then $\widetilde{£}$ is of second degree.

(iii) If the Carathéodory function $\mathbb{F}$ associated with $£$ has an analytic extension up to $|z|>r>1$, then the Carathéodory function $\widetilde{\mathbb{F}}$ of $\widetilde{£}$ has also an analytic extension to the exterior of the open unit disk.

Proof. If $£$ is a rational linear functional, and since $£_{\nu}$ is a rational linear functional, then the linear functional $\widetilde{f}$ is also rational. Therefore $\widetilde{\mathscr{E}}$ belongs to the Laguerre-Hahn affine class and it is also of second degree.

So let assume that $£$ is not a rational linear functional.

(i) If $£$ is a nonrational Laguerre-Hahn functional, then (see [2]), there exist polynomials $A \neq 0, B$, and $H$ such that

$$
A(z) \mathbb{F}^{\prime}(z)+B(z) \mathbb{F}(z)+H(z)=0, \quad \text { with } \quad z^{2} \bar{A}\left(\frac{1}{z}\right) B(z)+A(z) \bar{B}\left(\frac{1}{z}\right)=0 .
$$

Since $\widetilde{\mathbb{F}}(z)=\mathbb{F}(z)+\mathbb{F}_{v}(z)$, we can write $\widetilde{\mathbb{F}}(z)=\mathbb{F}(z)+P(z) / Q(z)$, with $P, Q$ polynomials of degree at most $k$ and hence the following differential equation holds

$$
A(z)\left(\widetilde{\mathbb{F}}^{\prime}(z)-\left(\frac{P(z)}{Q(z)}\right)^{\prime}\right)+B(z)\left(\widetilde{\mathbb{F}}(z)-\frac{P(z)}{Q(z)}\right)+H(z)=0,
$$

or, equivalently,

$$
A(z) \widetilde{\mathbb{F}}^{\prime}(z)+B(z) \widetilde{\mathbb{F}}(z)+H(z)-A(z) \frac{P^{\prime}(z) Q(z)-P(z) Q^{\prime}(z)}{Q^{2}(z)}-B(z) \frac{P(z)}{Q(z)}=0 .
$$

Therefore

$$
\begin{aligned}
& Q^{2}(z) A(z) \widetilde{\mathbb{F}}^{\prime}(z)+Q^{2}(z) B(z) \widetilde{\mathbb{F}}(z)+Q^{2}(z) H(z)-A(z)\left(P^{\prime}(z) Q(z)-P(z) Q^{\prime}(z)\right) \\
& \quad-B(z) P(z) Q(z)=0,
\end{aligned}
$$

that is, the formal series $\widetilde{\mathbb{F}}(z)$ satisfies the first-order linear differential equation

$$
\widetilde{A}(z) \widetilde{\mathbb{F}}^{\prime}(z)+\widetilde{B}(z) \widetilde{\mathbb{F}}(z)+\widetilde{H}(z)=0,
$$

where:

$$
\begin{aligned}
& \widetilde{A}(z)=Q^{2}(z) A(z), \\
& \widetilde{B}(z)=Q^{2}(z) B(z), \quad \text { and } \\
& \widetilde{H}(z)=Q^{2}(z) H(z)-A(z)\left(P^{\prime}(z) Q(z)-P(z) Q^{\prime}(z)\right)-B(z) P(z) Q(z) .
\end{aligned}
$$


Finally, since

$$
\begin{aligned}
z^{2} & \widetilde{\widetilde{A}}\left(\frac{1}{z}\right) \widetilde{B}(z)+A(z) \overline{\widetilde{B}}\left(\frac{1}{z}\right) \\
& =z^{2} \overline{Q^{2}}\left(\frac{1}{z}\right) \bar{A}\left(\frac{1}{z}\right) Q^{2}(z) B(z)+Q^{2}(z) A(z) \overline{Q^{2}}\left(\frac{1}{z}\right) \bar{B}\left(\frac{1}{z}\right) \\
& =\overline{Q^{2}}\left(\frac{1}{z}\right) Q^{2}(z)\left(z^{2} \bar{A}\left(\frac{1}{z}\right) B(z)+A(z) \bar{B}\left(\frac{1}{z}\right)\right)=0
\end{aligned}
$$

then (see [2]), the linear functional $\widetilde{£}$ belongs to the Laguerre-Hahn affine class.

(ii) If $£$ is a non rational and second degree linear functional then there exist polynomials $\alpha \neq 0, \beta$, and $\gamma \neq 0$ such that

$$
\alpha(z) \mathbb{F}^{2}(z)+\beta(z) \mathbb{F}(z)+\gamma(z)=0 \quad \text { and } \quad \beta^{2}-4 \alpha \gamma \neq r^{2},
$$

for every polynomial $r$. It is straightforward to prove that

$$
\alpha(z)\left(\widetilde{\mathbb{F}}(z)-\frac{P(z)}{Q(z)}\right)^{2}+\beta(z)\left(\widetilde{\mathbb{F}}(z)-\frac{P(z)}{Q(z)}\right)+\gamma(z)=0 .
$$

Thus

$$
\alpha(z) \widetilde{\mathbb{F}}^{2}(z)+\left(\beta(z)-2 \alpha(z) \frac{P(z)}{Q(z)}\right) \widetilde{\mathbb{F}}(z)+\gamma(z)-\frac{\beta(z) P(z)}{Q(z)}+\alpha(z)\left(\frac{P(z)}{Q(z)}\right)^{2}=0,
$$

and, as a consequence,

$$
\begin{aligned}
& Q^{2}(z) \alpha(z) \widetilde{\mathbb{F}}^{2}(z)+\left(Q^{2}(z) \beta(z)-2 \alpha(z) P(z) Q(z)\right) \widetilde{\mathbb{F}}(z) \\
& \quad+\gamma(z) Q^{2}(z)-\beta(z) P(z) Q(z)+\alpha(z) P^{2}(z)=0 .
\end{aligned}
$$

Thus the formal series $\widetilde{\mathbb{F}}(z)$ satisfies the quadratic equation

$$
\tilde{\alpha}(z) \widetilde{\mathbb{F}}^{2}(z)+\tilde{\beta}(z) \widetilde{\mathbb{F}}(z)+\tilde{\gamma}(z)=0,
$$

with

$$
\begin{aligned}
& \tilde{\alpha}(z)=Q^{2}(z) \alpha(z), \\
& \tilde{\beta}(z)=Q^{2}(z) \beta(z)-2 \alpha(z) P(z) Q(z), \\
& \tilde{\gamma}(z)=\gamma(z) Q^{2}(z)-\beta(z) P(z) Q(z)+\alpha(z) P^{2}(z) .
\end{aligned}
$$

Hence $\widetilde{\mathbb{F}}(z)$ is a second degree linear functional and

$$
\tilde{\beta}^{2}(z)-4 \tilde{\alpha}(z) \tilde{\gamma}(z)=\left(\beta^{2}(z)-4 \alpha(z) \gamma(z)\right) Q^{4}(z) \neq r^{2},
$$

for every polynomial $r$.

(iii) If we take into account that $\mathbb{F}_{v}(z)=\left(B_{k}(z) / B_{k}(0)\right)\left(1 / A_{k}(z)\right)$ and the fact that the zeros $\alpha_{j}$ of $A_{k}(z)$ are such that $\left|\alpha_{j}\right|>1$ for $j=1,2, \ldots, k$, then $\widetilde{F}(z)$ has an analytic extension up to $|z|>\min \left\{r,\left|\alpha_{j}\right|_{j=1,2, \ldots, k}\right\}>1$. 


\section{A particular case for measure $\mu$}

Now we consider the measure $\mu$ in relation (1) is the polynomial modification of the Lebesgue measure

$$
\mathrm{d} \mu(\theta)=\left|B_{k^{\prime}}\left(\mathrm{e}^{\mathrm{i} \theta}\right)\right|^{2} \frac{\mathrm{d} \theta}{2 \pi},
$$

where we assume that $B_{k^{\prime}}(z)$ is a polynomial of degree $k^{\prime} \geqslant 1$ and $B_{k^{\prime}}(0) \neq 0$. Then the measure $\tilde{\mu}$ considered in (1) becomes

$$
\mathrm{d} \tilde{\mu}(\theta)=\left|B_{k^{\prime}}\left(\mathrm{e}^{\mathrm{i} \theta}\right)\right|^{2} \frac{\mathrm{d} \theta}{2 \pi}+\frac{1}{\left|A_{k}\left(\mathrm{e}^{\mathrm{i} \theta}\right)\right|^{2}} \frac{\mathrm{d} \theta}{2 \pi} .
$$

Proposition 6. For each $n \geqslant k+k^{\prime}$ the polynomial $\psi_{n}(z)$ can be written in terms of, at most, $k+k^{\prime}+1$ polynomials $\left\{\tilde{\phi}_{j}(z)\right\}$, with $n-\left(k+k^{\prime}\right) \leqslant j \leqslant n$. That is, there exist $k+k^{\prime}$ complex numbers $\alpha_{n, n-1}, \alpha_{n, n-2}, \ldots, \alpha_{n, n-\left(k+k^{\prime}\right)}$ such that

$$
\psi_{n}(z)=\tilde{\phi}_{n}(z)+\alpha_{n, n-1} \tilde{\phi}_{n-1}(z)+\cdots+\alpha_{n, n-\left(k+k^{\prime}\right)} \tilde{\phi}_{n-\left(k+k^{\prime}\right)}(z) .
$$

Proof. Since for each $n$ the sequence of polynomials $\left\{\tilde{\phi}_{j}\right\}_{j=0}^{n}$ is a basis of the space $\mathbb{P}_{n}$ of polynomials of degree at most $n$, then we can write

$$
\psi_{n}(z)=\tilde{\phi}_{n}(z)+\sum_{j=0}^{n-1} \alpha_{n, j} \tilde{\phi}_{j}(z) .
$$

Therefore we have

$$
\left\langle\psi_{n}(z),\left.\tilde{\phi}_{j}(z)\right|_{\tilde{\mu}}=\alpha_{n, j}\left\|\tilde{\phi}_{j}\right\|_{\tilde{\mu}}^{2}, \quad j=1,2, \ldots, n-1 .\right.
$$

On the other hand, taking into account the definition of $\tilde{\mu}$ and since $\left\langle\psi_{n}(z), \tilde{\phi}_{j}(z)\right\rangle_{\nu}=0$ for $j=0,1, \ldots, n-1$, we get

$$
\alpha_{n, j}\left\|\tilde{\phi}_{j}\right\|_{\tilde{\mu}}^{2}=\left\langle\psi_{n}(z), \tilde{\phi}_{j}(z)\right\rangle_{\mu}, \quad \forall j=0,1,2, \ldots, n-1 .
$$

For $n \geqslant k+k^{\prime}$, since $\psi_{n}(z)=\left(1 / a_{k}\right) z^{n-k} A_{k}^{*}(z)$, then

$$
\left\langle\psi_{n}(z), \tilde{\phi}_{j}(z)\right\rangle_{\mu}=\frac{1}{a_{k}} \int_{0}^{2 \pi} z^{n-k} A_{k}^{*}(z) \overline{\tilde{\phi}_{j}(z)}\left|B_{k^{\prime}}(z)\right|^{2} \frac{\mathrm{d} \theta}{2 \pi} .
$$

Taking into account the form of the polynomials $A_{k}^{*}(z)$ and $B_{k^{\prime}}(z)$ it is easy to obtain

$$
\left\langle\psi_{n}(z), \tilde{\phi}_{j}(z)\right\rangle_{\mu}=0 \quad \text { for every } j=0,1, \ldots, n-\left(k+k^{\prime}\right)-1 .
$$

Hence $\alpha_{n, j}=0$ for every $j=0,1, \ldots, n-\left(k+k^{\prime}\right)-1$, and thus we get (17). 
Corollary 3. The coefficients in relation (17) satisfy the following properties:

(i) For $n>k+k^{\prime}$

$$
\alpha_{n, n-j}=\frac{\left\langle\psi_{n}(z), \tilde{\phi}_{n-j}(z)\right\rangle_{\mu}}{\left\|\tilde{\phi}_{n-j}\right\|_{\tilde{\mu}}^{2}}, \quad j=1,2, \ldots, k+k^{\prime} .
$$

(ii) For every $j=1,2, \ldots, k+k^{\prime}$

$$
\left|\alpha_{n, n-j}\right| \leqslant\left(\left|a_{k}\right|^{2}\left\|\psi_{n}\right\|_{\mu}^{2}+1\right)^{1 / 2} .
$$

(iii) Moreover, the coefficients satisfy the following inequalities

(a) For every $j=1,2, \ldots, k$,

$$
\left|\alpha_{n, n-j}-\frac{a_{k-j}}{a_{k}}\right| \leqslant\left(c_{0}(\mu)+c_{0}(v)\right)^{1 / 2} \sum_{l=0}^{j-1}\left|a_{k-l}\right| .
$$

(b) For every $j=k+1, k+2, \ldots, k+k^{\prime}$, we get

$$
\left|\alpha_{n, n-j}\right| \leqslant\left(c_{0}(\mu)+c_{0}(v)\right)^{1 / 2} \sum_{l=0}^{k}\left|a_{k-l}\right| .
$$

Proof. (i) (19) is a straightforward consequence of (18).

(ii) Since

$$
\begin{aligned}
\frac{\left|\left\langle\psi_{n}(z), \tilde{\phi}_{n-j}(z)\right\rangle_{\tilde{\mu}}\right|^{2}}{\left\|\tilde{\phi}_{n-j}\right\|_{\tilde{\mu}}^{4}} & \leqslant \frac{\left\|\psi_{n}\right\|_{\tilde{\mu}}^{2}\left\|\tilde{\phi}_{n-j}\right\|_{\tilde{\mu}}^{2}}{\left\|\tilde{\phi}_{n-j}\right\|_{\tilde{\mu}}^{4}}=\frac{\left\|\psi_{n}\right\|_{\tilde{\mu}}^{2}}{\left\|\tilde{\phi}_{n-j}\right\|_{\tilde{\mu}}^{2}}=\frac{\left\|\psi_{n}\right\|_{\mu}^{2}+\left\|\psi_{n}\right\|_{v}^{2}}{\left\|\tilde{\phi}_{n-j}\right\|_{\tilde{\mu}}^{2}} \\
& \leqslant \frac{\left\|\psi_{n}\right\|_{\mu}^{2}+1 /\left|a_{k}\right|^{2}}{\left\|\psi_{n-j}\right\|_{v}^{2}}=\frac{\left\|\psi_{n}\right\|_{\mu}^{2}+1 /\left|a_{k}\right|^{2}}{1 /\left|a_{k}\right|^{2}}=\left|a_{k}\right|^{2}\left\|\psi_{n}\right\|_{\mu}^{2}+1
\end{aligned}
$$

and $\left\langle\psi_{n}(z), \tilde{\phi}_{n-j}(z)\right\rangle_{\tilde{\mu}}=\left\langle\psi_{n}(z), \tilde{\phi}_{n-j}(z)\right\rangle_{\mu}$ for every $j \geqslant 1$, then we deduce

$$
\left|\alpha_{n, n-j}\right|=\frac{\left|\left\langle\psi_{n}(z), \tilde{\phi}_{n-j}(z)\right\rangle_{\tilde{\mu}}\right|}{\left\|\tilde{\phi}_{n-j}\right\|_{\tilde{\mu}}^{2}} .
$$

Hence (20) is obtained.

(iii) (a) For each $j=1,2, \ldots, k$, we get

$$
\begin{aligned}
\alpha_{n, n-j} & =\frac{\left\langle\psi_{n}(z), \tilde{\phi}_{n-j}(z)\right\rangle_{\tilde{\mu}}}{\left\|\tilde{\phi}_{n-j}\right\|_{\tilde{\mu}}^{2}} \\
& =\frac{\left(a_{k-j} / a_{k}\right)\left\langle z^{n-j}, \tilde{\phi}_{n-j}(z)\right\rangle_{\tilde{\mu}}+\sum_{l=0}^{j-1}\left(a_{k-l} / a_{k}\right)\left\langle z^{n-l}, \tilde{\phi}_{n-j}(z)\right\rangle_{\tilde{\mu}}}{\left\|\tilde{\phi}_{n-j}\right\|_{\tilde{\mu}}^{2}} \\
& =\frac{a_{k-j}}{a_{k}}+\frac{\sum_{l=0}^{j-1}\left(a_{k-l} / a_{k}\right)\left\langle z^{n-l}, \tilde{\phi}_{n-j}(z)\right\rangle_{\tilde{\mu}}}{\left\|\tilde{\phi}_{n-j}\right\|_{\tilde{\mu}}^{2}} .
\end{aligned}
$$


Thus

$$
\left|\alpha_{n, n-j}-\frac{a_{k-j}}{a_{k}}\right| \leqslant \sum_{l=0}^{j-1} \frac{\left|a_{k-l}\right|}{\left|a_{k}\right|} \frac{\left|\left\langle z^{n-l}, \tilde{\phi}_{n-j}(z)\right\rangle_{\tilde{\mu}}\right|}{\left\|\tilde{\phi}_{n-j}\right\|_{\tilde{\mu}}^{2}} .
$$

Taking into account that for each $l=1,2, \ldots, j-1$ it holds

$$
\begin{aligned}
\frac{\left|\left\langle z^{n-l}, \tilde{\phi}_{n-j}(z)\right\rangle_{\tilde{\mu}}\right|^{2}}{\left\|\tilde{\phi}_{n-j}\right\|_{\tilde{\mu}}^{4}} & \leqslant \frac{\left\|z^{n-l}\right\|_{\tilde{\mu}}^{2}\left\|\tilde{\phi}_{n-j}\right\|_{\tilde{\mu}}^{2}}{\left\|\tilde{\phi}_{n-j}\right\|_{\tilde{\mu}}^{4}}=\frac{\left\|z^{n-l}\right\|_{\tilde{\mu}}^{2}}{\left\|\tilde{\phi}_{n-j}\right\|_{\tilde{\mu}}^{2}} \\
& =\frac{\left\|z^{n-l}\right\|_{\mu}^{2}+\left\|z^{n-l}\right\|_{v}^{2}}{\left\|\tilde{\phi}_{n-j}\right\|_{\mu}^{2}+\left\|\tilde{\phi}_{n-j}\right\|_{\nu}^{2}} \leqslant \frac{c_{0}(\mu)+c_{0}(v)}{\left\|\phi_{n-j}\right\|_{\mu}^{2}+\left\|\psi_{n-j}\right\|_{v}^{2}} \\
& \leqslant \frac{c_{0}(\mu)+c_{0}(v)}{m(\mu)+1 /\left|a_{k}\right|^{2}} \leqslant \frac{c_{0}(\mu)+c_{0}(v)}{1 /\left|a_{k}\right|^{2}}=\left(c_{0}(\mu)+c_{0}(v)\right)\left|a_{k}\right|^{2},
\end{aligned}
$$

then

$$
\frac{\left|\left\langle z^{n-l}, \tilde{\phi}_{n-j}(z)\right\rangle_{\tilde{\mu}}\right|}{\left\|\tilde{\phi}_{n-j}\right\|_{\tilde{\mu}}^{2}} \leqslant\left(c_{0}(\mu)+c_{0}(v)\right)^{1 / 2}\left|a_{k}\right| .
$$

Substitution in (23) yields (21).

(b) For $j=k+1, k+2, \ldots, k+k^{\prime}$ we have

$$
\begin{aligned}
\alpha_{n, n-j} & =\frac{\left\langle\psi_{n}(z), \tilde{\phi}_{n-j}(z)\right\rangle_{\tilde{\mu}}}{\left\|\tilde{\phi}_{n-j}\right\|_{\tilde{\mu}}^{2}}=\frac{\left\langle z^{n}+\left(a_{k-1} / a_{k}\right) z^{n-1}+\cdots+\left(a_{0} / a_{k}\right) z^{n-k}, \tilde{\phi}_{n-j}(z)\right\rangle_{\tilde{\mu}}}{\left\|\tilde{\phi}_{n-j}\right\|_{\tilde{\mu}}^{2}} \\
& =\sum_{l=0}^{k} \frac{a_{k-l}}{a_{k}} \frac{\left\langle z^{n-l}, \tilde{\phi}_{n-j}(z)\right\rangle_{\tilde{\mu}}}{\left\|\tilde{\phi}_{n-j}\right\|_{\tilde{\mu}}^{2}}
\end{aligned}
$$

which implies

$$
\left|\alpha_{n, n-j}\right| \leqslant \sum_{l=0}^{k} \frac{\left|a_{k-l}\right| \mid}{\left|a_{k}\right|} \frac{\left|\left\langle z^{n-l}, \tilde{\phi}_{n-j}(z)\right\rangle_{\tilde{\mu}}\right|}{\left\|\tilde{\phi}_{n-j}\right\|_{\tilde{\mu}}^{2}}, \quad j=k+1, k+2, \ldots, k+k^{\prime} .
$$

From

$$
\frac{\left|\left\langle z^{n-l}, \tilde{\phi}_{n-j}(z)\right\rangle_{\tilde{\mu}}\right|^{2}}{\left\|\tilde{\phi}_{n-j}\right\|_{\tilde{\mu}}^{4}} \leqslant \frac{\left\|z^{n-l}\right\|_{\tilde{\mu}}^{2}\left\|\tilde{\phi}_{n-j}\right\|_{\tilde{\mu}}^{2}}{\left\|\tilde{\phi}_{n-j}\right\|_{\tilde{\mu}}^{4}} \leqslant \frac{c_{0}(\mu)+c_{0}(v)}{\left\|\psi_{n-j}\right\|_{\nu}^{2}} \leqslant\left|a_{k}\right|^{2}\left(c_{0}(\mu)+c_{0}(v)\right),
$$

we obtain

$$
\frac{\left|\left\langle z^{n-l}, \tilde{\phi}_{n-j}(z)\right\rangle_{\tilde{\mu}}\right|}{\left\|\tilde{\phi}_{n-j}\right\|_{\tilde{\mu}}^{2}} \leqslant\left|a_{k}\right|\left(c_{0}(\mu)+c_{0}(v)\right)^{1 / 2}, \quad j=k+1, k+2, \ldots, k+k^{\prime},
$$

and substituting in (24) we have (22).

Corollary 4. The Szegö function $\Pi(z ; \tilde{\mu})$ of measure $\tilde{\mu}$ is a rational transformation of the Szegö function $\Pi(z ; v)$ corresponding to the measure $v$. 
Proof. From relation (17), we have, for each $n \geqslant k+k^{\prime}$

$$
z^{n}+\sum_{j=1}^{k} \frac{a_{k-j}}{a_{k}} z^{n-k}=\tilde{\phi}_{n}(z)+\sum_{j=1}^{k+k^{\prime}} \alpha_{n, n-j} \tilde{\phi}_{n-j}(z) .
$$

By identifying the coefficients of $z^{n-1}$ we obtain

$$
\frac{a_{k-1}}{a_{k}}=\frac{\tilde{\phi}_{n}^{(n-1)}(0)}{(n-1) !}+\alpha_{n, n-1}, \quad \forall n \geqslant k+k^{\prime},
$$

that is,

$$
\alpha_{n, n-1}=\frac{a_{k-1}}{a_{k}}-\frac{\tilde{\phi}_{n}^{(n-1)}(0)}{(n-1) !}, \quad \forall n \geqslant k+k^{\prime} .
$$

Taking limits and using that $\mu \in \mathbb{S}$ we get that the sequence of coefficients $\left\{\alpha_{n, n-1}\right\}$ is convergent. Thus we denote its $\operatorname{limit}_{\text {by }} \lim _{n \rightarrow \infty} \alpha_{n, n-1}=\mathcal{B}_{1}$.

Proceeding in the same way with the coefficients of $z^{n-j}$ with $j=2,3, \ldots, k+k^{\prime}$, we get

$$
\frac{a_{k-j}}{a_{k}}=\frac{\tilde{\phi}_{n}^{(n-j)}(0)}{(n-j) !}+\sum_{i=1}^{j-1} \alpha_{n, n-i} \frac{\tilde{\phi}_{n-i}^{(n-j)}(0)}{(n-i) !}+\alpha_{n, n-j}, \quad 2 \leqslant j \leqslant k,
$$

and

$$
0=\frac{\tilde{\phi}_{n}^{(n-j)}(0)}{(n-j) !}+\sum_{i=1}^{j-1} \alpha_{n, n-i} \frac{\tilde{\phi}_{n-i}^{(n-j)}(0)}{(n-i) !}+\alpha_{n, n-j}, \quad k+1 \leqslant j \leqslant k+k^{\prime} .
$$

Hence there exists $\lim _{n \rightarrow \infty} \alpha_{n, n-j}=\mathcal{B}_{j}$ for each $j=1,2, \ldots, k+k^{\prime}$.

We divide by $z^{n}$ in relation (17)

$$
\frac{\psi_{n}(z)}{z^{n}}=\frac{\tilde{\phi}_{n}(z)}{z^{n}}+\sum_{j=1}^{k+k^{\prime}} \frac{\alpha_{n, n-j}}{z^{j}} \frac{\tilde{\phi}_{n-j}(z)}{z^{n-j}},
$$

and take into account that for compact subsets of $|z|>1$ it holds

$$
\lim _{n \rightarrow \infty} \frac{\psi_{n}(z)}{z^{n}}=\frac{\bar{\Pi}(1 / z ; v)}{\overline{\Pi(0 ; \nu)}} \quad \text { and } \quad \lim _{n \rightarrow \infty} \frac{\tilde{\phi}_{n}(z)}{z^{n}}=\frac{\bar{\Pi}(1 / z ; \tilde{\mu})}{\overline{\Pi(0 ; \tilde{\mu})}} .
$$

Therefore, if we take limits then we obtain

$$
\frac{\bar{\Pi}(1 / z ; v)}{\overline{\Pi(0 ; v)}}=\left(1+\sum_{j=1}^{k+k^{\prime}} \frac{\mathcal{B}_{j}}{z^{j}}\right) \frac{\bar{\Pi}(1 / z ; \tilde{\mu})}{\overline{\Pi(0 ; \tilde{\mu})}},
$$

which yields our result. 


\subsection{An example}

Let us consider the measure defined as a sum of the polynomial modification of the Lebesgue measure $\mathrm{d} \mu(\theta)=\left|\mathrm{e}^{\mathrm{i} \theta}-\beta\right|^{2} \mathrm{~d} \theta /(2 \pi)$, with $\beta \neq 0$, and the rational modification of the Lebesgue measure $\mathrm{d} v(\theta)=\left(1 /\left|\mathrm{e}^{\mathrm{i} \theta}-\alpha\right|^{2}\right) \mathrm{d} \theta /(2 \pi)$, with $|\alpha|<1$ and $\alpha \neq 0$, that is

$$
\begin{aligned}
\mathrm{d} \tilde{\mu}(\theta) & =\mathrm{d} \mu(\theta)+\mathrm{d} \nu(\theta) \\
& =\left|\mathrm{e}^{\mathrm{i} \theta}-\beta\right|^{2} \frac{\mathrm{d} \theta}{2 \pi}+\frac{1}{\left|\mathrm{e}^{\mathrm{i} \theta}-\alpha\right|^{2}} \frac{\mathrm{d} \theta}{2 \pi}, \quad|\alpha|<1, \alpha \neq 0, \text { and } \beta \neq 0 .
\end{aligned}
$$

We denote by $\left\{c_{n}(v)\right\}_{n=0}^{\infty}$ the sequence of moments of measure $v$

$$
c_{n}(\nu)=\int_{0}^{2 \pi} \mathrm{e}^{\mathrm{i} n \theta} \mathrm{d} \nu(\theta)=\frac{\alpha^{n}}{1-|\alpha|^{2}}, \quad \forall n \geqslant 0 .
$$

In the sequel we assume that the measure is normalized, and therefore the moments are

$$
c_{n}(v)=\alpha^{n}, \quad \forall n \geqslant 0 .
$$

We also denote by $\left\{c_{n}(\mu)\right\}_{n=0}^{\infty}$ the moments of the measure $\mu$, that is, $c_{0}(\mu)=1+|\beta|^{2}$, $c_{1}(\mu)=-\beta$, and $c_{n}(\mu)=0 \quad \forall n \geqslant 2$. We also assume that the measure is normalized, and thus

$$
c_{0}(\mu)=1, \quad c_{1}(\mu)=\frac{-\beta}{1+|\beta|^{2}}, \quad c_{n}(\mu)=0 \quad \forall n \geqslant 2 .
$$

It is very well known that (see [10])

- $\psi_{n}(z)=z^{n-1}(z-\alpha)$, for $n \geqslant 1$.

- $(z-\beta) \phi_{n}(z)=z^{n+1}-\left(\beta^{n+1} / K_{n}(\beta, \beta)\right) K_{n}(z, \beta)$, for $n \geqslant 0$, where $K_{n}(z, \beta)=$ $\sum_{j=0}^{n} z^{j} \bar{\beta}^{j}$ and $K_{n}(\beta, \beta)=\sum_{j=0}^{n}|\beta|^{2 j}$.

- The norms satisfy the following relations:

(1) $\left\|\psi_{n}\right\|_{v}^{2}=1-|\alpha|^{2}$ for $n \geqslant 1$, and $\left\|\psi_{0}\right\|_{v}^{2}=1$.

$$
\left\|\phi_{n}\right\|_{\mu}^{2}=\frac{1}{1+|\beta|^{2}}\left(1+\frac{|\beta|^{2(n+1)}}{\sum_{j=0}^{n}|\beta|^{2 j}}\right) \text { for every } n \geqslant 0 .
$$

(3) $m(v)=\lim _{n \rightarrow \infty}\left\|\psi_{n}\right\|_{v}^{2}=1-|\alpha|^{2}$ and

$$
m(\mu)=\lim _{n \rightarrow \infty}\left\|\phi_{n}\right\|_{\mu}^{2}= \begin{cases}\frac{1}{1+|\beta|^{2}}, & \text { if }|\beta| \leqslant 1 \\ \frac{|\beta|^{2}}{1+|\beta|^{2}}, & \text { if }|\beta|>1 .\end{cases}
$$


The moments of the measure $\tilde{\mu}$ can be obtained as $c_{n}(\tilde{\mu})=c_{n}(\mu)+c_{n}(\nu)$ and therefore

$$
c_{0}(\tilde{\mu})=2, \quad c_{1}(\tilde{\mu})=\frac{\alpha-\beta+\alpha|\beta|^{2}}{1+|\beta|^{2}} \quad \text { and } \quad c_{n}(\tilde{\mu})=\alpha^{n}, \quad \forall n \geqslant 2 .
$$

We also consider the normalized sequence of moments, that is,

$$
c_{0}(\tilde{\mu})=1, \quad c_{1}(\tilde{\mu})=\frac{\alpha-\beta+\alpha|\beta|^{2}}{2\left(1+|\beta|^{2}\right)} \quad \text { and } \quad c_{n}(\tilde{\mu})=\frac{\alpha^{n}}{2}, \quad \forall n \geqslant 2 .
$$

(Notice that an interesting case is obtained when $\alpha=1 / 2$ and $\beta=1$. In this situation the moments are $c_{0}(\tilde{\mu})=1, c_{1}(\tilde{\mu})=0$, and $c_{n}(\tilde{\mu})=1 / 2^{n+1}, \forall n \geqslant 2$.)

Proposition 7. The polynomials $\left\{\psi_{n}\right\}_{n=0}^{\infty}$ and $\left\{\tilde{\phi}_{n}\right\}_{n=0}^{\infty}$ are related, for $n \geqslant 2$, by

$$
\begin{aligned}
\psi_{n}(z)= & \tilde{\phi}_{n}(z)+\frac{-(\alpha+\beta)+\alpha \beta\left(\tilde{\phi}_{n-1}^{(n-2)}(0) /(n-2) !-\bar{\beta}\right)}{\left(1+|\beta|^{2}\right)\left\|\tilde{\phi}_{n-1}\right\|_{\tilde{\mu}}^{2}} \tilde{\phi}_{n-1}(z) \\
& +\frac{\alpha \beta}{\left(1+|\beta|^{2}\right)\left\|\tilde{\phi}_{n-2}\right\|_{\tilde{\mu}}^{2}} \tilde{\phi}_{n-2}(z) .
\end{aligned}
$$

Proof. In this case, for $k=k^{\prime}=1$, relation (17) becomes

$$
\psi_{n}(z)=\tilde{\phi}_{n}(z)+\alpha_{n, n-1} \tilde{\phi}_{n-1}(z)+\alpha_{n, n-2} \tilde{\phi}_{n-2}(z) .
$$

We obtain the coefficients taking into account (19) and the following relations

$$
\begin{aligned}
\left\langle\psi_{n}(z), \tilde{\phi}_{n-1}(z)\right\rangle_{\mu} & =\frac{-(\alpha+\beta)+\alpha \beta\left(\tilde{\phi}_{n-1}^{(n-2)}(0) /(n-2) !-\bar{\beta}\right)}{1+|\beta|^{2}}, \\
\left\langle\psi_{n}(z), \tilde{\phi}_{n-2}(z)\right\rangle_{\mu} & =\frac{\alpha \beta}{1+|\beta|^{2}} .
\end{aligned}
$$

Finally, by substitution we get (26).

Corollary 5. It holds that

(i) For each $n \geqslant 2$, the polynomials $\tilde{\phi}_{n}(z)$ are determined by the polynomials $\tilde{\phi}_{n-1}(z)$, $\tilde{\phi}_{n-2}(z)$, and their norms as follows

$$
\begin{aligned}
\tilde{\phi}_{n}(z)= & z^{n-1}(z-\alpha)-\frac{-(\alpha+\beta)+\alpha \beta\left(\tilde{\phi}_{n-1}^{(n-2)}(0) /(n-2) !-\bar{\beta}\right)}{\left\|\tilde{\phi}_{n-1}\right\|_{\tilde{\mu}}^{2}} \tilde{\phi}_{n-1}(z) \\
& -\frac{\alpha \beta}{\left\|\tilde{\phi}_{n-2}\right\|_{\tilde{\mu}}^{2}} \tilde{\phi}_{n-2}(z) .
\end{aligned}
$$

(ii) $\tilde{\phi}_{1}(z)=z-\left(\alpha-\beta+\alpha|\beta|^{2}\right) /\left(2\left(1+|\beta|^{2}\right)\right)$. 
(iii) (a) The limit of the squares of the norms, $m(\tilde{\mu})$, satisfies the quadratic equation

$$
\begin{aligned}
& (m(\tilde{\mu}))^{2}-\left(1+\frac{\bar{\alpha} \beta+\alpha \bar{\beta}}{2\left(1+|\beta|^{2}\right)}\right) m(\tilde{\mu}) \\
& \quad+\left(|\alpha \beta|^{2}+|-(\alpha+\beta)+\alpha \beta(A-\bar{\beta})|^{2}\right)=0, \quad \text { with } A=\lim _{n \rightarrow \infty} \frac{\tilde{\phi}_{n-1}^{(n-2)}(0)}{(n-2) !} .
\end{aligned}
$$

(b) Moreover, it holds

$$
m(\mu)+1 \leqslant m(\tilde{\mu}) \leqslant 1+\frac{1+|\alpha+\beta|^{2}+|\alpha \beta|^{2}}{1+|\beta|^{2}} .
$$

Proof. (i) To obtain (27), it suffices to take into account the form of the polynomials $\psi_{n}(z)$ and relation (26).

(ii) It is immediate since $c_{0}(\tilde{\mu})=1$ and $c_{1}(\tilde{\mu})=\left(\alpha-\beta+\alpha|\beta|^{2}\right) /\left(2\left(1+|\beta|^{2}\right)\right)$.

(iii) (a) We take $\tilde{\mu}$ norms in (27)

$$
\left\|z^{n-1}(z-\alpha)\right\|_{\tilde{\mu}}^{2}=\left\|\tilde{\phi}_{n}\right\|_{\tilde{\mu}}^{2}+\frac{\left|-(\alpha+\beta)+\alpha \beta\left(\tilde{\phi}_{n-1}^{(n-2)}(0) /(n-2) !-\bar{\beta}\right)\right|^{2}}{\left\|\tilde{\phi}_{n-1}\right\|_{\tilde{\mu}}^{2}}+\frac{|\alpha \beta|^{2}}{\left\|\tilde{\phi}_{n-2}\right\|_{\tilde{\mu}}^{2}} .
$$

Since $\left\|z^{n-1}(z-\alpha)\right\|_{\tilde{\mu}}^{2}=1+(\bar{\alpha} \beta+\alpha \bar{\beta}) /\left(2\left(1+|\beta|^{2}\right)\right)$, if we take limits in the preceding equality when $n \rightarrow \infty$ and use that $\tilde{\mu} \in \mathbb{S}$, then it follows that there exists

$$
\lim _{n \rightarrow \infty} \frac{\tilde{\phi}_{n-1}^{(n-2)}(0)}{(n-2) !}=A \text {. }
$$

Therefore

$$
m(\tilde{\mu})=1+\frac{\bar{\alpha} \beta+\alpha \bar{\beta}}{2\left(1+|\beta|^{2}\right)}-\frac{|-(\alpha+\beta)+\alpha \beta(A-\bar{\beta})|^{2}}{m(\tilde{\mu})}-\frac{|\alpha \beta|^{2}}{m(\tilde{\mu})},
$$

and (28) follows.

(b) Relation (29) is evident if we take into account (3), $a_{k}=1$, and

$$
\|z-\alpha\|_{\mu}^{2}=\frac{1+|\alpha+\beta|^{2}+|\alpha \beta|^{2}}{1+|\beta|^{2}} .
$$

\section{The case of a particular Bernstein-Szegö measure}

Let us consider now that the Borel positive measure $\tilde{\mu}$ is defined by

$$
\mathrm{d} \tilde{\mu}(\theta)=\mathrm{d} \mu(\theta)+\frac{1}{\left|\mathrm{e}^{\mathrm{i} \theta}-\alpha\right|^{2}} \frac{\mathrm{d} \theta}{2 \pi},
$$

with $0<|\alpha|<1$, and $\mu$ a Borel positive measure. We denote, as before, by $\left\{\phi_{n}\right\}_{n=0}^{\infty}$ the $\operatorname{SMOP}(\mu)$ in the standard sense. 
We also denote by $\left\{\hat{\phi}_{n}\right\}_{n=0}^{\infty}$ the $\operatorname{SMOP}(\hat{\mu})$, where $\hat{\mu}$ is the polynomial modification of the measure $\mu, \mathrm{d} \hat{\mu}(\theta)=\left|\mathrm{e}^{\mathrm{i} \theta}-\alpha\right|^{2} \mathrm{~d} \mu(\theta)$, and by $\left\{\tilde{\phi}_{n}\right\}_{n=0}^{\infty}$ the $\operatorname{SMOP}(\tilde{\mu})$.

Proposition 8. The $\operatorname{SMOP}(\tilde{\mu})$ is given by

$$
\tilde{\phi}_{n+1}(z)=\phi_{n+1}(z)-\mathbb{K}_{n}^{\mathrm{T}}(z)\left(\left(1-|\alpha|^{2}\right) \tilde{M}_{n}^{-1}+\mathcal{K}_{n}\right)^{-1} \Phi_{n+1},
$$

with

$$
\begin{aligned}
\mathbb{K}_{n}^{\mathrm{T}}(z) & =\left(K_{n}(z, \alpha), \mathbb{K}_{n}^{\mathrm{T}}(z, 0)\right) \\
& =\left(K_{n}(z, \alpha), K_{n}(z, 0), K_{n}^{(0,1)}(z, 0), \ldots, K_{n}^{(0, n)}(z, 0)\right),
\end{aligned}
$$

where

$$
K_{n}(z, y)=\sum_{j=0}^{n} \frac{\phi_{j}(z) \overline{\phi_{j}(y)}}{\left\|\phi_{j}\right\|_{\mu}^{2}}
$$

is the $n$-reproducing polynomial kernel associated with the measure $\mu$;

$$
\widetilde{M}_{n}=\left(\begin{array}{c|c}
1 \mid & 0 \\
\hline 0 \mid D M_{n} \widetilde{D}
\end{array}\right), \quad M_{n}=\left(\begin{array}{cccc}
\alpha-\frac{1}{\bar{\alpha}} & \alpha^{2}-\frac{1}{\bar{\alpha}^{2}} & \ldots & \alpha^{n+1}-\frac{1}{\bar{\alpha}^{n+1}} \\
0 & \alpha-\frac{1}{\bar{\alpha}} & \ldots & \alpha^{n}-\frac{1}{\bar{\alpha}^{n}} \\
\vdots & \vdots & \ddots & \vdots \\
0 & 0 & \ldots & \alpha^{2}-\frac{1}{\bar{\alpha}^{2}} \\
0 & 0 & \ldots & \alpha-\frac{1}{\bar{\alpha}}
\end{array}\right),
$$

where

$$
\begin{gathered}
D=\operatorname{diag}\left(1, \frac{1}{1 !}, \ldots, \frac{1}{n !}\right), \quad \widetilde{D}=\operatorname{diag}\left(\frac{1}{1 !}, \frac{1}{2 !}, \ldots, \frac{1}{(n+1) !}\right) ; \\
\mathcal{K}_{n}=\left(\begin{array}{c|c}
K_{n}\left(\frac{1}{\bar{\alpha}}, \alpha\right) & \mathbb{K}_{n}^{\mathrm{T}}\left(\frac{1}{\bar{\alpha}}, 0\right) \\
\hline \mathbb{K}_{n}^{\prime}(0, \alpha) & \left(K_{n}^{(i+1, j)}(0,0)\right)_{\substack{i=0, \ldots, n \\
j=0, \ldots, n}}^{(i+1, j}
\end{array}\right),
\end{gathered}
$$

and

$$
\widetilde{\Phi}_{n+1}=\left(\tilde{\phi}_{n+1}\left(\frac{1}{\bar{\alpha}}\right), \widetilde{\Phi}_{n+1}^{\mathrm{T}}(0)\right)=\left(\tilde{\phi}_{n+1}\left(\frac{1}{\bar{\alpha}}\right), \tilde{\phi}_{n+1}^{(1)}(0), \tilde{\phi}_{n+1}^{(2)}(0), \ldots, \tilde{\phi}_{n+1}^{(n+1)}(0)\right) .
$$

Proof. Taking into account that an orthogonal basis of $\mathbb{P}_{n}$ is

$$
\left\{K_{n}(z, \alpha)\right\} \cup\left\{(z-\alpha) \hat{\phi}_{j}(z)\right\}_{j=0}^{n-1},
$$


(see [5]), we have

$$
\tilde{\phi}_{n+1}(z)=\phi_{n+1}(z)+\lambda_{n, n} K_{n}(z, \alpha)+\sum_{j=0}^{n-1} \lambda_{n, j}(z-\alpha) \hat{\phi}_{j}(z) .
$$

Notice that for $0 \leqslant j \leqslant n-1$,

$$
\begin{aligned}
\lambda_{n, j} & =\frac{1}{\left\|\hat{\phi}_{j}\right\|_{\hat{\mu}}^{2}} \int_{\mathbb{T}} \tilde{\phi}_{n+1}(y) \overline{(y-\alpha)} \overline{\hat{\phi}_{j}(y)} \mathrm{d} \mu(y) \text { and } \\
\lambda_{n, n} & =\frac{\tilde{\phi}_{n+1}(\alpha)-\phi_{n+1}(\alpha)}{K_{n}(\alpha, \alpha)} .
\end{aligned}
$$

Therefore

$$
\tilde{\phi}_{n+1}(z)=\phi_{n+1}(z)+\lambda_{n, n} K_{n}(z, \alpha)+(z-\alpha) \int_{\mathbb{T}} \tilde{\phi}_{n+1}(y) \overline{(y-\alpha)} \widehat{K}_{n-1}(z, y) \mathrm{d} \mu(y),
$$

where $\widehat{K}_{n-1}(z, y)$ is the $(n-1)$-reproducing polynomial kernel of the measure $\hat{\mu}$.

In the last integral we substitute $\mathrm{d} \mu(y)$ by $\mathrm{d} \tilde{\mu}(y)-\left(1 /|y-\alpha|^{2}\right) \mathrm{d} \theta /(2 \pi)$, and we apply the orthogonality of $\left\{\tilde{\phi}_{n}\right\}_{n=0}^{\infty}$. Hence we obtain

$$
\tilde{\phi}_{n+1}(z)=\phi_{n+1}(z)+\lambda_{n, n} K_{n}(z, \alpha)-\int_{\mathbb{T}} \tilde{\phi}_{n+1}(y) \frac{(z-\alpha) \overline{(y-\alpha)}}{|y-\alpha|^{2}} \widehat{K}_{n-1}(z, y) \frac{\mathrm{d} \theta}{2 \pi} .
$$

On the other hand, if we take into account the following result (see [5])

$$
(z-\alpha) \overline{(y-\alpha)} \widehat{K}_{n-1}(z, y)=K_{n}(z, y)-\frac{K_{n}(z, \alpha) K_{n}(\alpha, y)}{K_{n}(\alpha, \alpha)},
$$

then the substitution in (34) yields

$$
\begin{aligned}
\tilde{\phi}_{n+1}(z)= & \phi_{n+1}(z)+\lambda_{n, n} K_{n}(z, \alpha) \\
& -\int_{\mathbb{T}} \frac{\tilde{\phi}_{n+1}(y)}{|y-\alpha|^{2}}\left(K_{n}(z, y)-\frac{K_{n}(z, \alpha) K_{n}(\alpha, y)}{K_{n}(\alpha, \alpha)}\right) \frac{\mathrm{d} \theta}{2 \pi} .
\end{aligned}
$$

Since

$$
\lambda_{n, n}=\frac{1}{K_{n}(\alpha, \alpha)} \int_{\mathbb{T}} \tilde{\phi}_{n+1}(y) K_{n}(\alpha, y) \mathrm{d} \mu(y)=-\frac{1}{K_{n}(\alpha, \alpha)} \int_{\mathbb{T}} \tilde{\phi}_{n+1}(y) \frac{K_{n}(\alpha, y)}{|y-\alpha|^{2}} \frac{\mathrm{d} \theta}{2 \pi},
$$

from (35) it follows that

$$
\begin{aligned}
\tilde{\phi}_{n+1}(z) & =\phi_{n+1}(z)-\int_{\mathbb{T}} \frac{\tilde{\phi}_{n+1}(y) K_{n}(z, y)}{|y-\alpha|^{2}} \frac{\mathrm{d} \theta}{2 \pi} \\
& =\phi_{n+1}(z)-\frac{1}{2 \pi \mathrm{i}} \int_{\mathbb{T}} \frac{\tilde{\phi}_{n+1}(y) K_{n}(z, y)}{(y-\alpha)(1-\bar{\alpha} y)} \mathrm{d} y .
\end{aligned}
$$


Taking conjugates in the last integral and applying the residue theorem we get

$$
\begin{aligned}
& \frac{1}{2 \pi \mathrm{i}} \int_{\mathbb{T}} \frac{\tilde{\phi}_{n+1}^{*}(y) K_{n}(y, z)}{(y-\alpha)(1-\bar{\alpha} y) y^{n+1}} \mathrm{~d} y \\
& \quad=\frac{\tilde{\phi}_{n+1}^{*}(\alpha) K_{n}(\alpha, z)}{\left(1-|\alpha|^{2}\right) \alpha^{n+1}}+\left.\frac{1}{n !} \frac{\mathrm{d}^{n}}{\mathrm{~d} y^{n}}\left(\frac{\tilde{\phi}_{n+1}^{*}(y) K_{n}(y, z)}{(y-\alpha)(1-\bar{\alpha} y)}\right)\right|_{y=0} \\
& \quad=\frac{\overline{\tilde{\phi}}_{n+1}(1 / \alpha)}{1-|\alpha|^{2}} K_{n}(\alpha, z)+\left.\frac{1}{n !} \sum_{k=0}^{n}\left(\begin{array}{l}
n \\
k
\end{array}\right) K_{n}^{(k, 0)}(0, z)\left(\frac{\tilde{\phi}_{n+1}^{*}(y)}{(y-\alpha)(1-\bar{\alpha} y)}\right)^{(n-k)}\right|_{y=0} .
\end{aligned}
$$

On the other hand, we have

$$
\begin{aligned}
& \left.\left(\frac{\tilde{\phi}_{n+1}^{*}(y)}{(y-\alpha)(1-\bar{\alpha} y)}\right)^{(n-k)}\right|_{y=0} \\
& \quad=\left.\left.\sum_{j=0}^{n-k}\left(\begin{array}{c}
n-k \\
j
\end{array}\right)\left(\tilde{\phi}_{n+1}^{*}(y)\right)^{(j)}\right|_{y=0}\left(\frac{1}{y-\alpha}+\frac{\bar{\alpha}}{1-\bar{\alpha} y}\right)^{(n-k-j)}\right|_{y=0} \frac{1}{1-|\alpha|^{2}} \\
& =\left.\frac{1}{1-|\alpha|^{2}} \sum_{j=0}^{n-k}\left(\begin{array}{c}
n-k \\
j
\end{array}\right)\left(\tilde{\phi}_{n+1}^{*}(y)\right)^{(j)}\right|_{y=0}(n-k-j) !\left(\bar{\alpha}^{n-k-j+1}-\left(\alpha^{-1}\right)^{n-k-j+1}\right),
\end{aligned}
$$

and taking into account that if

$$
\tilde{\phi}_{n+1}(y)=y^{n+1}+a_{n+1, n} y^{n}+\cdots+a_{n+1,0},
$$

then

$$
\tilde{\phi}_{n+1}^{*}(y)=1+\bar{a}_{n+1, n} y+\cdots+\bar{a}_{n+1,0} y^{n+1},
$$

we have, for $j=0,1, \ldots, n+1,\left.\left(\tilde{\phi}_{n+1}^{*}(y)\right)^{(j)}\right|_{y=0}=j ! \bar{a}_{n+1, n+1-j}$.

Hence, substitution in (38) yields

$$
\begin{aligned}
& \left.\left(\frac{\tilde{\phi}_{n+1}^{*}(y)}{(y-\alpha)(1-\tilde{\alpha} y)}\right)^{(n-k)}\right|_{y=0} \\
& \quad=\frac{1}{1-|\alpha|^{2}} \sum_{j=0}^{n-k}\left(\begin{array}{c}
n-k \\
j
\end{array}\right) j !(n-k-j) ! \bar{a}_{n+1, n+1-j}\left(\bar{\alpha}^{n-k-j+1}-\left(\alpha^{-1}\right)^{n-k-j+1}\right) \\
& \quad=\frac{(n-k) !}{1-|\alpha|^{2}} \sum_{j=0}^{n-k} \bar{a}_{n+1, n+1-j}\left(\bar{\alpha}^{n-k-j+1}-\left(\alpha^{-1}\right)^{n-k-j+1}\right)
\end{aligned}
$$


Therefore, substituting this last expression in (37) we get

$$
\begin{aligned}
& \frac{1}{2 \pi \mathrm{i}} \int_{\mathbb{T}} \frac{\tilde{\phi}_{n+1}^{*}(y) K_{n}(y, z)}{(y-\alpha)(1-\bar{\alpha} y) y^{n+1}} \mathrm{~d} y \\
& =\frac{\tilde{\tilde{\phi}}_{n+1}(1 / \alpha)}{1-|\alpha|^{2}} K_{n}(\alpha, z) \\
& \quad+\frac{1}{1-|\alpha|^{2}} \sum_{k=0}^{n} \frac{1}{k !} \overline{K_{n}^{(0, k)}(z, 0)} \sum_{j=0}^{n-k} \frac{\overline{\tilde{\phi}_{n+1}^{(n+1)}(0)}}{(n+1-j) !}\left(\bar{\alpha}^{n-k-j+1}-\left(\alpha^{-1}\right)^{n-k-j+1}\right) .
\end{aligned}
$$

Inserting this expression in (36) we get

$$
\begin{aligned}
\tilde{\phi}_{n+1}(z)= & \phi_{n+1}(z)-\frac{\tilde{\phi}_{n+1}(1 / \bar{\alpha})}{1-|\alpha|^{2}} K_{n}(z, \alpha)-\frac{1}{1-|\alpha|^{2}} \sum_{k=0}^{n} \frac{1}{k !} K_{n}^{(0, k)}(z, 0) \\
& \times\left(\sum_{j=0}^{n-k} \frac{\tilde{\phi}_{n+1}^{(n+1-j)}(0)}{(n+1-j) !}\left(\alpha^{n-k-j+1}-\left(\bar{\alpha}^{-1}\right)^{n-k-j+1}\right)\right) .
\end{aligned}
$$

Using a matrix notation, this last formula can be represented in the following more simple form:

$$
\tilde{\phi}_{n+1}(z)=\phi_{n+1}(z)-\frac{\tilde{\phi}_{n+1}(1 / \bar{\alpha})}{1-|\alpha|^{2}} K_{n}(z, \alpha)-\frac{1}{1-|\alpha|^{2}} \mathbb{K}_{n}^{\mathrm{T}}(z, 0) D M_{n} \widetilde{D} \widetilde{\Phi}_{n+1}(0),
$$

where

$$
\begin{aligned}
\mathbb{K}_{n}^{\mathrm{T}}(z, 0) & =\left(K_{n}(z, 0), K_{n}^{(0,1)}(z, 0), \ldots, K_{n}^{(0, n)}(z, 0)\right), \\
\widetilde{\Phi}_{n+1}^{\mathrm{T}}(0) & =\left(\tilde{\phi}_{n+1}^{(1)}(0), \tilde{\phi}_{n+1}^{(2)}(0), \ldots, \tilde{\phi}_{n+1}^{(n+1)}(0)\right), \\
D & =\operatorname{diag}\left(1, \frac{1}{1 !}, \ldots, \frac{1}{n !}\right), \quad \widetilde{D}=\operatorname{diag}\left(\frac{1}{1 !}, \frac{1}{2 !}, \ldots, \frac{1}{(n+1) !}\right),
\end{aligned}
$$

and $M_{n}$ is given in (31), or, equivalently

$$
\tilde{\phi}_{n+1}(z)=\phi_{n+1}(z)-\frac{1}{1-|\alpha|^{2}} \mathbb{K}_{n}^{\mathrm{T}}(z) \widetilde{M}_{n} \widetilde{\Phi}_{n+1},
$$

with

$$
\mathbb{K}_{n}^{\mathrm{T}}(z)=\left(K_{n}(z, \alpha), \mathbb{K}_{n}^{\mathrm{T}}(z, 0)\right), \quad \widetilde{\Phi}_{n+1}=\left(\tilde{\phi}_{n+1}\left(\frac{1}{\bar{\alpha}}\right), \widetilde{\Phi}_{n+1}^{\mathrm{T}}(0)\right),
$$

and $\widetilde{M}_{n}$ is given in (31).

If we evaluate (40) at $z=1 / \bar{\alpha}$ then we obtain

$$
\tilde{\phi}_{n+1}\left(\frac{1}{\bar{\alpha}}\right)=\phi_{n+1}\left(\frac{1}{\bar{\alpha}}\right)-\frac{1}{1-|\alpha|^{2}} \mathbb{K}_{n}^{\mathrm{T}}\left(\frac{1}{\bar{\alpha}}\right) \widetilde{M}_{n} \widetilde{\Phi}_{n+1}
$$


On the other hand, if we take derivatives up to the $j$ th order in (40) and evaluate at $z=0$, then we get

$$
\tilde{\phi}_{n+1}^{(j)}(0)=\phi_{n+1}^{(j)}(0)-\frac{1}{1-|\alpha|^{2}}\left(\mathbb{K}_{n}^{\mathrm{T}}\right)^{(j)}(0) \widetilde{M}_{n} \widetilde{\Phi}_{n+1}, \quad \text { for } j=1,2, \ldots, n+1,
$$

or, equivalently, using a vector notation

$$
\widetilde{\Phi}_{n+1}=\Phi_{n+1}-\frac{1}{1-|\alpha|^{2}} \mathcal{K}_{n} \widetilde{M}_{n} \widetilde{\Phi}_{n+1},
$$

where $\mathcal{K}_{n}$ is given by (32).

Therefore

$$
\widetilde{\Phi}_{n+1}=\left(I+\frac{1}{1-|\alpha|^{2}} \mathcal{K}_{n} \widetilde{M}_{n}\right)^{-1} \Phi_{n+1},
$$

and, thus (40) becomes

$$
\tilde{\phi}_{n+1}(z)=\phi_{n+1}(z)-\frac{1}{1-|\alpha|^{2}} \mathbb{K}_{n}^{\mathrm{T}}(z) \tilde{M}_{n}\left(I+\frac{1}{1-|\alpha|^{2}} \mathcal{K}_{n} \widetilde{M}_{n}\right)^{-1} \Phi_{n+1} .
$$

Finally, since $\widetilde{M}_{n}$ is nonsingular, this last expression can be rewritten as (30).

Remark 1. Notice that

1. The rows of the matrix $\mathcal{K}_{n}$ are evaluations of the vector $\mathbb{K}_{n}^{\mathrm{T}}(z)$ at $1 / \bar{\alpha}$ and evaluations of the derivatives from order 1 up to $n+1$ at $z=0$. Therefore, the entries of the last row are zeros.

2. $\Phi_{n+1}=\left(\Phi_{n+1}(1 / \bar{\alpha}), \Phi_{n+1}^{\mathrm{T}}(0)\right)$.

3. In a preceding paper [1] we have obtained the explicit expression of $K_{n}^{(i, j)}(0,0)$.

4. The reasoning about the nonsingularity of the matrix $\left(1-|\alpha|^{2}\right) \tilde{M}_{n}^{-1}+\mathcal{K}_{n}$ is the same as in [1].

\section{Acknowledgement}

The authors thank the referees for their valuable remarks and suggestions in order to improve our manuscript.

\section{References}

[1] A. Cachafeiro, F. Marcellán, and C. Pérez, Lebesgue perturbation of a quasi-definite hermitian functional. The positive definite case, Linear Algebra Appl. 369 (2003) 235-250.

[2] A. Cachafeiro and C. Pérez, A study of the Laguerre-Hahn affine functionals on the unit circle, J. Comput. Anal. Appl. (2004). 
[3] L. Daruis, P. González Vera, and F. Marcellán, Gaussian quadrature formulae on the unit circle, J. Comput. Appl. Math. 140 (2002) 159-183.

[4] S. Elhay, G.H. Golub, and J. Kautsky, Jacobi matrices for sums of weight functions, BIT 32 (1992) 144-166.

[5] P. García-Lázaro and F. Marcellán, Christoffel formulas for $n$-kernels associated to Jordan arcs, in: Orthogonal Polynomials and Applications, ed. C. Brezinski et al., Lecture Notes in Mathematics, Vol. 1171 (Springer, New York, 1985) pp. 195-203.

[6] Y.L. Geronimus, Polynomials Orthogonal on a Circle and Their Applications, American Mathematical Society Translations Series 1, Vol. 3 (Amer. Math. Soc., Providence, RI, 1962) pp. 1-78.

[7] U. Grenander and G. Szegö, Toeplitz. Forms and Their Applications (Univ. of California Press, Berkeley, 1958).

[8] T. Kailath and B. Porat, State-space generators for orthogonal polynomials, in: Prediction Theory and Harmonic Analysis, eds. V. Mandrekar and H. Salehi (North-Holland, Amsterdam, 1983) pp. 131163.

[9] V.F. Pisarenko, The retrieval of harmonics from a covariance function, Geophys. J. Royal Austral. Soc. 33 (1973) 347-366.

[10] G. Szegö, Orthogonal Polynomials, 4th ed., American Mathematics Society Colloquium Publications, Vol. 23 (Amer. Math. Soc., Providence, RI, 1975). 